\title{
Uso do Auxílio Emergencial por adultos com diabetes tipo 1 durante a pandemia de
} COVID-19 no Brasil, 2020

\author{
Use of Financial Emergency Aid for adults with type 1 diabetes during COVID-19 pandemic in
}

Brazil, 2020

Uso de Ayuda de Emergencia por adultos con diabetes tipo 1 durante la pandemia de COVID-19 en

Brasil, 2020

\section{Resumo}

Objetivo: Buscou-se analisar o uso do auxílio emergencial na aquisição de insumos para o tratamento por adultos com Diabetes Tipo 1 durante o distanciamento social pela pandemia de COVID-19 no Brasil. Métodos: Pesquisa transversal, realizada em julho de 2020, no Brasil, através de um formulário online sobre dados socioeconômicos, demográficos, monitorização glicêmica e aquisição de insumos. Foi aplicado o teste Qui-Quadrado de Pearson, com análise de resíduos ajustados $(\mathrm{p}<0,05)$. Resultados: Participaram 472 adultos, dos quais 39,19\% relataram ter recebido o auxílio emergencial do Governo Federal. Receber o auxílio estava associado a não ter ensino superior $(\mathrm{p}<0,000)$, morar em bairros de periferia $(\mathrm{p}<0,000)$, ter tido a renda reduzida durante a pandemia $(\mathrm{p}=0,001)$ e ter renda familiar de até 2 salários mínimos ( $\mathrm{p}=0,000)$. A maioria usou o auxílio para o pagamento de contas básicas $(57,5 \%)$ e compra de alimentos $(43,8 \%)$. Conclusões: Usar o auxílio para comprar insumos do tratamento estava associado a adquiri-los com dinheiro próprio ou por doação e não gastar o auxílio com insumos estava associado a adquiri-los por meio do Sistema Único de Saúde (SUS). O auxílio emergencial foi utilizado para aquisição de insumos essenciais ao tratamento e necessidades básicas, especialmente em pessoas com piores condições socioeconômicas e que não conseguiram adquirir estes materiais pelo SUS.

Palavras-chave: Diabetes Tipo 1; Pandemias; Glicemia.

\section{Abstract}

Objective: We sought to analyze the use of financial emergency aid in the acquisition of inputs for treatment by adults with Type 1 Diabetes during social distancing due to the pandemic of COVID-19 in Brazil. Method: Cross-sectional survey carried out during July 2020, in Brazil, using an online form about socioeconomic, demographic, glycemic 
monitoring and input acquisition. Pearson's Chi-Square test was applied, with adjusted residual analysis $(\mathrm{p}<0.05)$. Results: 472 adults participated, of which 39.19\% reported having received emergency financial aid from the Federal Government. Receiving the aid was associated with not having higher education $(\mathrm{p}<0.000)$, living in peripheral neighborhoods $(p<0.000)$, having reduction in income during the pandemic $(p=0.001)$ and having a family income of up to 2 minimum wages ( $\mathrm{p}=0.000)$. Most participants used the aid to pay basic bills $(57.5 \%)$ and purchase food (43.8\%). Conclusion: Using the aid to buy treatment supplies was associated with purchasing them with their own money or by donation and not spending the aid with supplies was associated with purchasing them through the Unified Health System (Sistema Único de Saúde - SUS). Financial emergency aid was used to purchase essential supplies for treatment and basic needs, especially for people with worse socioeconomic conditions and who were unable to acquire these materials through SUS.

Keywords: Type 1 Diabetes; Pandemics; Glycemic.

\section{Resumen}

Objetivo: Buscamos analizar el uso de la ayuda de emergencia en la adquisición de insumos para el tratamiento de adultos con Diabetes tipo 1 durante el distanciamiento social debido a la pandemia de COVID-19 en Brasil. Métodos: Encuesta transversal, realizada en julio de 2020, en Brasil, mediante formulario en línea sobre datos socioeconómicos y demográficos, seguimiento glucémico y adquisición de insumos. Se aplicó la prueba Chi-Cuadrado de Pearson, con análisis de residuos ajustados $(\mathrm{p}<0,05)$. Resultados: Participaron 472 adultos, de los cuales 39,19\% reportaron haber recibido asistencia de emergencia por parte del Gobierno Federal. Recibir la ayuda se asoció con no tener estudios superiores $(p<0,000)$, vivir en barrios periféricos $(p<0,000)$, haber tenido ingresos reducidos durante la pandemia $(\mathrm{p}=0,001)$ y tener un ingreso familiar de hasta 2 salarios mínimos $(\mathrm{p}=0,000)$. La mayoría usó la ayuda para pagar las cuentas básicas $(57,5 \%)$ y comprar alimentos $(43,8 \%)$. Conclusiones: Utilizar la ayuda para comprar insumos de tratamiento se asoció con adquirirlos con dinero propio o por donación, y no gastar la ayuda en insumos se asoció con adquirirlos a través del Sistema Único de Salud (SUS). La ayuda de emergencia se utilizó para comprar suministros esenciales para el tratamiento y las necesidades básicas, especialmente para personas con peores condiciones socioeconómicas y que no podían adquirir estos materiales a través del SUS.

Palabras clave: Diabetes Tipo 1; Pandemias; Glucemia.

\section{Introdução}

A principal estratégia para diminuir as taxas de contágio na pandemia provocada pelo SARS-CoV-2 (COVID-19) é o distanciamento social, no entanto, grande parte da população não consegue aderir a esta recomendação sem ter impacto importante em sua condição socioeconômica (Barreto et al., 2020). A partir de 2015, houve uma redução do crescimento econômico brasileiro e um aumento da taxa de desemprego, resultando em uma maior vulnerabilidade socioeconômica (IBGE, 2021; Carvalho et al, 2021). Com a chegada da pandemia, as taxas de desemprego sofreram maior aumento, chegando a $14,9 \%$ no terceiro trimestre de 2020 e no primeiro trimestre de 2021, e muitos trabalhadores informais perderam sua renda (IBGE, 2021). Para estas pessoas, existe uma necessidade de garantir que condições básicas de sobrevivência, como a alimentação e a saúde, sejam garantidas por políticas públicas (Costa, 2020). Desta forma, os programas de transferência de renda são essenciais para diminuir a vulnerabilidade socioeconômica e garantir a proteção social (Arrais et al., 2020).

No Brasil, os programas de transferência de renda integraram-se na Política Nacional de Assistência Social, com o objetivo de reduzir as desigualdades sociais (Córdova \& Alves, 2019). Durante a pandemia, houve a criação do Auxílio Emergencial (Lei $\mathrm{n}^{\mathrm{o}}$ 13.982/2020) (Brasil, 2020), inicialmente por três meses, entretanto, o recebimento foi prorrogado diversas vezes, sendo a última prorrogação realizada em março de 2021 (Brasil, 2021).

Dados da última Pesquisa de Orçamentos Familiares (POF) (Instituto Brasileiro de Geografia e Estatística - IBGE, 2019) que, em média, $11,5 \%$ da renda dos brasileiros se destina aos custos relacionados à assistência à saúde, medicamentos e plano/seguro de saúde. Quando se refere às doenças crônicas, este percentual pode aumentar, como é o caso do Diabetes Mellitus tipo 1 (DM1), o qual, segundo a Sociedade Brasileira de Diabetes (SBD), tem incidência de 7,6 casos por 100 mil indivíduos no Brasil, com destaque para o aumento desta incidência em crianças menores de 5 anos (SBD, 2019). Não foram encontrados na literatura dados sobre a prevalência de DM1 em adultos brasileiros, entretanto, dados da International Diabetes Federation (IDF), em 2021, indicaram que 15,7 milhões de adultos entre 20 e 79 anos vivem com diabetes no Brasil, sem diferenciar a prevalência de Diabetes tipo 1 e 2. 
Segundo Cobas et al., (2013), o custo médio por paciente com DM1 para o Sistema Único de Saúde (SUS) era cerca de US\$1319,35 e as maiores despesas estavam relacionadas às insulinas e insumos para monitorização glicêmica, ambos indispensáveis para o tratamento. Entretanto, este valor já está defasado, não havendo novos estudos em âmbito nacional sobre os gastos do paciente com DM1 com seu tratamento.

No Brasil, há uma lei que regulamenta o fornecimento de insumos, através do SUS, para o manejo da glicemia para os pacientes com diabetes, incluindo tiras reagentes, lancetas e insulinas (Brasil, 2006; Ministério da Saúde, 2006). Entretanto, mesmo antes da pandemia, observava-se inconsistência no fornecimento desses materiais (Andrade \& Alves, 2014; Souza et al., 2018), levando os pacientes a comprar esses insumos com a própria renda, o que em um contexto pandêmico torna-se mais difícil.

Considerando que o descontrole glicêmico é um dos fatores que pode contribuir para o agravamento das complicações da COVID-19 (Holman et al., 2020), é possível que haja uma relação bastante estreita entre vulnerabilidade socioeconômica e risco de morbimortalidade por COVID-19 em pessoas com diabetes. Para adultos com DM1 com renda familiar prejudicada durante a pandemia, o auxílio emergencial poderá ser usado para adquirir os insumos do tratamento caso ocorram inadequações no fornecimento pelo SUS. Dessa forma, o fator socioeconômico pode estar intimamente relacionado à adesão ao tratamento e, consequentemente, ao melhor controle glicêmico e prevenção de complicações clínicas associadas.

Portanto, o presente estudo teve como objetivo analisar o uso do auxílio emergencial na aquisição de insumos para o tratamento de adultos com DM1 durante o período de distanciamento social pela pandemia de COVID-19 no Brasil.

\section{Metodologia}

Estudo transversal, descritivo e analítico, realizado com uma amostra por conveniência, executado após aprovação pelo Comitê de Ética e Pesquisa (Parecer 4.047.909), conforme as resoluções no 466/2012 e 510/2016 (Brasil, 2012; 2016).

Um convite contendo o link com o formulário on-line da pesquisa e o Termo de Consentimento Livre e Esclarecido (TCLE), foi divulgado pelas mídias sociais de um Projeto de Extensão de uma universidade pública do Pará (Brasil). A pesquisa foi realizada em julho de 2020, período em que vigoravam restrições de distanciamento social em todos os estados brasileiros.

Foram considerados como critérios de inclusão: ter diagnóstico de DM1, idade $\geq 18$ anos e aceitar participar da pesquisa escolhendo a opção "Li o TCLE e aceito participar da pesquisa" disponível no formulário. Os participantes deveriam escolher qual a sua condição, dentre: ser adulto com DM1, não ter diabetes, ter outros tipos de diabetes, ser criança/adolescente com DM1, ser cuidador. Caso a seleção não fosse correspondente ao público de adultos com DM1, a pesquisa encerrava-se automaticamente. Foram excluídos aqueles que selecionaram alguma alternativa que não condizia com os critérios de inclusão, que não concluíram a pesquisa ou não concordaram com o TCLE. A participação no estudo foi voluntária e anônima.

Foi desenvolvido um formulário por meio da plataforma Formulários Google ${ }^{\circledR}$, no formato de pesquisa de opinião (Brasil, 2016). As perguntas foram divididas nos seguintes eixos:

a. Sociodemográfico: sexo biológico; idade; escolaridade; região; tipo de cidade (capital, região metropolitana ou interior do estado); tipo de bairro (favela ou comunidade, periferia, classe média, classe alta ou zona rural e a opção "nenhuma das anteriores" foi retirada, pois condizia a não saber informar seu tipo de bairro).

b. Situação financeira: renda familiar em Salários Mínimos (SM) (valor vigente em 2020 de R \$1.045), em menor que 1; entre 1 e 2; entre 3 até 5; acima de 5 até 10; acima de 10 até 20; maior que 20; recebimento do auxílio emergencial pelo participante ou por algum membro da sua residência (sim, recebeu o auxílio; não, porque ninguém se enquadrava nos critérios; não, mesmo se enquadrando nos critérios); caso tenha recebido auxílio emergencial, utilizou para pagar ou adquirir quais itens (contas; cartão de crédito; roupas; itens para casa; alimentos; fitas ou lancetas; seringas ou agulhas; insulina ou 
hipoglicemiante; outros materiais relacionados ao DM; remédios; outros), podendo nesta última pergunta escolher mais de uma alternativa.

c. Monitorização da glicemia: instrumento utilizado para a monitorização glicêmica (glicosímetro; Sistema Flash de Monitorização da Glicose [SFMG]; SFMG e glicosímetro; ou se não monitorava a glicemia).

d. Aquisição de insumos (considerando os 30 dias anteriores à pesquisa): como adquiriu os insumos (insulinas; tiras reagentes; lancetas; ou materiais necessários para o SFMG) para o tratamento do DM1 (por meio do SUS; comprou com dinheiro próprio; recebeu doação; não precisou adquirir; não conseguiu adquirir; outro; ou não usa este insumo).

Foi utilizado o software SPSS, versão 21. Os resultados descritivos foram expressos em média, desvio padrão ou frequência absoluta e proporção para as variáveis categóricas. Para a estatística analítica, foi aplicado o teste Qui-Quadrado de Pearson com análise de resíduos ajustados (nível de significância estatística de p<0,05).

\section{Resultados}

Participaram da pesquisa 472 adultos com média de idade de 30,24 anos $( \pm 9,75), 86 \%$ eram do sexo feminino, a maioria residia na região Sudeste (47,0\%), 52,97\% tinham ensino superior completo ou em curso. Quanto à renda familiar mensal, 32,42\% recebiam de três a cinco SM, 39,19\% alegaram ter recebido o auxílio emergencial, 54\% relataram não precisar do auxílio e 6,77\% não haviam recebido apesar de solicitar. Observou-se associação entre receber o auxílio emergencial e não ter ensino superior $(p<0,000)$, morar em bairros de periferia $(p<0,000)$, ter a renda reduzida na pandemia $(p=0,001)$ e ter renda familiar menor que 1 SM até $2 \mathrm{SM}(\mathrm{p}=0,000)$ (Tabela 1$)$.

Não ter recebido o auxílio emergencial apresentou associação com idade maior ou igual a 45 anos ( $\mathrm{p}=0,033)$, ensino superior $(\mathrm{p}<0,000)$, morar no Centro-Oeste $(\mathrm{p}=0,019)$ e em bairro de classe alta $(\mathrm{p}<0,000)$, ter renda maior ou igual anteriormente $(\mathrm{p}=0,001)$ e ter renda familiar de 5 até $20 \mathrm{SM}(\mathrm{p}=0,000)$ (Tabela 1$)$. 
Tabela 1. Caracterização da amostra de adultos com Diabetes Tipo 1 de acordo com os aspectos socioeconômicos e recebimento do Auxílio Emergencial em período de distanciamento social pela pandemia de COVID-19 no Brasil, 2020.

\begin{tabular}{|c|c|c|c|c|}
\hline & $\begin{array}{c}\text { Recebeu o auxílio } \\
\text { n }(\%)\end{array}$ & $\begin{array}{c}\text { Não recebeu o auxílio } \\
\text { n (\%) }\end{array}$ & $\begin{array}{l}\text { Total } \\
\text { n }(\%) \\
\end{array}$ & p-valor* \\
\hline \multicolumn{5}{|l|}{ Faixa Etária } \\
\hline 18 a 24 anos & $70(14,8)$ & $91(19,3)$ & $161(34,1)$ & \multirow{3}{*}{0,033 T } \\
\hline 25 a 44 anos & $106(22,5)$ & $163(34,5)$ & $269(57,0)$ & \\
\hline Maior ou igual a 45 anos & $9(1,9)$ & $33(7,0)^{(+)}$ & $42(8,9)$ & \\
\hline \multicolumn{5}{|l|}{ Escolaridade } \\
\hline Sem Ensino Superior & $59(12,5)^{(+)}$ & $43(9,1)^{(-)}$ & $102(22,1)$ & \multirow{2}{*}{$<0,000$ I } \\
\hline Com Ensino Superior & $126(26,7)^{(-)}$ & $244(51,6)^{(+)}$ & $370(77,9)$ & \\
\hline \multicolumn{5}{|l|}{ Sexo } \\
\hline Feminino & $162(34,3)$ & $244(51,7)$ & $406(86,0)$ & \multirow{2}{*}{0,435} \\
\hline Masculino & $23(4,9)$ & $43(9,1)$ & $66(14,0)$ & \\
\hline \multicolumn{5}{|l|}{ Regiãa } \\
\hline Norte & $17(3,6)$ & $16(3,4)$ & $33(7,0)$ & \multirow{5}{*}{0,019 T } \\
\hline Nordeste & $46(9,7)$ & $51(10,8)$ & $97(20,6)$ & \\
\hline Centro-oeste & $8(1,7)^{(-)}$ & $29(6,1)^{(+)}$ & $37(7,8)$ & \\
\hline Sudeste & $88(18,6)$ & $134(28,4)$ & $222(47,0)$ & \\
\hline Sul & $26(5,5)$ & $57(2,1)$ & $83(17,6)$ & \\
\hline \multicolumn{5}{|l|}{ Bairro } \\
\hline Favela ou Comunidade & $9(1,9)^{(+)}$ & $1(0,2)^{(-)}$ & $10(2,1)$ & \multirow{5}{*}{$<0,000$ T } \\
\hline Periferia & $40(8,5)^{(+)}$ & $39(8,3)^{(-)}$ & $79(16,7)$ & \\
\hline Classe Média & $96(20,3)$ & $164(34,7)$ & $260(55,1)$ & \\
\hline Classe Alta & $13(2,8)^{(-)}$ & $60(12,7)^{(+)}$ & $73(15,5)$ & \\
\hline Zona Rural & $8(1,7)$ & $12(2,5)$ & $20(4,2)$ & \\
\hline \multicolumn{5}{|l|}{ Cidade } \\
\hline Capital ou Região Metropolitana & $110(23,3)$ & $185(39,2)$ & $295(62,5)$ & \multirow{2}{*}{0,273} \\
\hline Interior do Estado & $75(15,9)$ & $102(21,6)$ & $177(37,5)$ & \\
\hline \multicolumn{5}{|l|}{ Manutenção da Renda } \\
\hline Renda $\geq$ que antes da Pandemia & $81(17,2)^{(-)}$ & $170(36,0)^{(+)}$ & $251(53,2)$ & \multirow{2}{*}{$0,001 \Phi$} \\
\hline Renda $<$ que antes da Pandemia & $104(22,0)^{(+)}$ & $117(24,8)^{(-)}$ & $221(46,8)$ & \\
\hline \multicolumn{5}{|l|}{ Renda Familiar** } \\
\hline Menor que $1 \mathrm{SM}$ & $16(3,4)^{(+)}$ & $3(0,6)^{(-)}$ & $19(4,0)$ & \multirow{6}{*}{$0,000 \mathrm{~T}$} \\
\hline Entre 1 a 2 SM & $86(12,8)^{(+)}$ & $48(10,2)^{(-)}$ & $134(28,4)$ & \\
\hline Entre 3 a $5 \mathrm{SM}$ & $52(11,0)$ & $101(21,4)$ & $153(32,4)$ & \\
\hline Entre 5 e $10 \mathrm{SM}$ & $18(3,8)^{(-)}$ & $85(18,0)^{(+)}$ & $103(21,8)$ & \\
\hline Entre 10 a $20 \mathrm{SM}$ & $10(2,1)^{(-)}$ & $36(7,6)^{(+)}$ & $46(9,7)$ & \\
\hline Mais de $20 \mathrm{SM}$ & $3(0,6)$ & $14(3,0)$ & $17(3,6)$ & \\
\hline
\end{tabular}

*Teste de Qui-quadrado; **Salário Mínimo = R\$1.045,00; † Estatística significativa; Análise de resíduos: (+) Associação significativa, (-) Associação significativa negativa. Nota: foram retirados os dados referentes aos participantes que assinalaram "Nenhuma das opções". Fonte: Dados da pesquisa (2020).

Dentre os que receberam o auxílio emergencial $(\mathrm{n}=185)$, 59,7\% usaram para pagar contas básicas, 57,5\% compraram alimentos e 43,8\% adquiriram medicamentos. Em relação à aquisição de insumos para manejo da glicemia, 24,2\% adquiriram fitas ou lancetas com o auxílio emergencial, 22,7\% outros insumos, 22,0\% insulinas ou hipoglicemiantes e 17,7\% compraram seringas ou agulhas (Tabela 2). 
Tabela 2. Uso do auxílio emergencial por adultos com Diabetes Tipo 1 em período de distanciamento social pela pandemia de COVID-19 no Brasil, 2020.

\begin{tabular}{lc}
\hline \multicolumn{1}{c}{ Uso do auxílio } & n (\%) \\
\hline Contas básicas & $111(59,7 \%)$ \\
Cartão de crédito & $46(24,9 \%)$ \\
Roupas & $5(2,7 \%)$ \\
Itens para casa & $28(15,1 \%)$ \\
Alimentos & $107(57,5 \%)$ \\
Fitas ou lancetas & $45(24,2 \%)$ \\
Insulina ou Hipoglicemiantes & $41(22 \%)$ \\
Seringas ou Agulhas & $33(17,7 \%)$ \\
Outros materiais para o DM & $42(22,7 \%)$ \\
Remédios & $81(43,8 \%)$ \\
Outros & $39(21 \%)$ \\
\hline
\end{tabular}

Fonte: Dados da pesquisa (2020).

Receber o auxílio emergencial foi associado à maneira que adquiriu lancetas $(\mathrm{p}=0,031)$. Adquirir lancetas por meio do SUS estava associado a ter recebido o auxílio e inversamente associado a não ter recebido por não ter os critérios. Adquirir lancetas com dinheiro próprio estava inversamente associado a receber o auxílio e associado a não ter recebido por não ter os requisitos. Houve associação inversa entre não ter precisado adquirir lancetas anteriormente e não ter recebido auxílio mesmo com os critérios (Tabela 3).

Houve associação entre a aquisição de SFMG e o recebimento de auxílio emergencial (p<0,000), em que adquirir o SFMG por meio do SUS ou comprar com dinheiro próprio estava associado inversamente a ter recebido o auxílio e diretamente a não ter recebido o auxílio, mesmo se enquadrando nos critérios. Não usar SFMG estava associado a receber o auxílio (Tabela 3). 
Tabela 3. Associação entre a aquisição de insumos para manejo da glicemia e o recebimento de Auxílio Emergencial por adultos com Diabetes Tipo 1 em período de distanciamento social pela pandemia de COVID-19 no Brasil, 2020.

\begin{tabular}{|c|c|c|c|c|}
\hline \multirow[b]{2}{*}{ Aquisição de insumos } & \multicolumn{3}{|c|}{ Recebimento do auxílio emergencial } & \multirow[b]{2}{*}{ p-valor* } \\
\hline & $\begin{array}{c}\text { Sim, recebi o auxílio. } \\
\text { n }(\%)\end{array}$ & $\begin{array}{c}\text { Não, ninguém se } \\
\text { enquadrava nos critérios. } \\
n(\%)\end{array}$ & $\begin{array}{l}\text { Não, mesmo tendo } \\
\text { critérios. } \\
\text { n }(\%)\end{array}$ & \\
\hline \multicolumn{5}{|l|}{ Insulina } \\
\hline Pelo SUS & $114(24,2)$ & $144(30,5)$ & $21(4,4)$ & \multirow{6}{*}{0,652} \\
\hline Dinheiro próprio & $53(11,2)$ & $92(19,5)$ & $8(1,7)$ & \\
\hline Doação & $5(1,1)$ & $6(1,3)$ & $2(0,4)$ & \\
\hline Não precisou adquirir & $5(1,1)$ & $6(1,3)$ & $1(0,2)$ & \\
\hline Não consegui adquirir & $1(0,2)$ & 0 & 0 & \\
\hline Outros & $7(1,5)$ & $7(1,5)$ & 0 & \\
\hline \multicolumn{5}{|l|}{ Tiras Reagentes } \\
\hline Pelo SUS & $111(23,8)$ & $154(33)$ & $24(5,1)$ & \multirow{7}{*}{0,347} \\
\hline Dinheiro próprio & $47(10,1)$ & $71(15,2)$ & $7(1,5)$ & \\
\hline Doação & $9(1,9)$ & $10(2,1)$ & 0 & \\
\hline Não precisou adquirir & $2(0,4)$ & $10(2,1)$ & 0 & \\
\hline Não consegui adquirir & $5(1,1)$ & $2(0,4)$ & 0 & \\
\hline Outros & $6(1,3)$ & $3(0,6)$ & $1(0,2)$ & \\
\hline Não uso glicosímetro & $2(0,4)$ & $3(0,6)$ & $0(0)$ & \\
\hline \multicolumn{5}{|l|}{ Lancetas } \\
\hline Pelo SUS & $98(21,1)^{(+)}$ & $101(21,7)^{(-)}$ & $19(4,1)$ & \multirow{7}{*}{$0,031 \Phi$} \\
\hline Dinheiro próprio & $44(9,5)^{(-)}$ & $96(20,6)^{(+)}$ & $8(1,7)$ & \\
\hline Doação & $11(2,4)$ & $11(2,3)$ & $4(0,9)$ & \\
\hline Não precisou adquirir & $17(3,7)$ & $32(6,9)$ & $0^{(-)}$ & \\
\hline Não consegui adquirir & $1(0,2)$ & $1(0,2)$ & 0 & \\
\hline Outros & $8(1,7)$ & $6(1,3)$ & $1(0,2)$ & \\
\hline Não uso glicosímetro & $3(0,6)$ & $4(0,9)$ & 0 & \\
\hline \multicolumn{5}{|l|}{$S F M G^{* *}$} \\
\hline Pelo SUS & $5(1,3)^{(-)}$ & $15(4)$ & $6(1,6)^{(+)}$ & \multirow{7}{*}{$<0,000$ Ф } \\
\hline Dinheiro próprio & $22(5,9)^{(-)}$ & $70(18,8)^{(+)}$ & $5(1,3)$ & \\
\hline Doação & $6(1,6)$ & $7(1,9)$ & $1(0,3)$ & \\
\hline Não precisou adquirir & $2(0,5)$ & $2(0,5)$ & $0(0)$ & \\
\hline Não consegui adquirir & $1(0,3)$ & $3(0,8)$ & $0(0)$ & \\
\hline Outros & $3(0,8)$ & $9(2,4)$ & $0(0)$ & \\
\hline Não uso & $119(31,9)^{(+)}$ & $86(23,1)^{(-)}$ & $11(2,9)^{(-)}$ & \\
\hline
\end{tabular}

*Teste de Qui-quadrado; **Sistema Flash de Monitorização da Glicose; T Estatística significativa; † Análise de resíduos: (+) Associação significativa, (-) Associação significativa negativa. Nota ${ }^{1}$ : $\mathrm{O}$ nde participantes inferior na tabela em relação ao $\mathrm{n}$ total da pesquisa é em decorrência de alguns participantes não terem respondido essas perguntas no formulário. Nota ${ }^{2}$ : A soma do percentual ultrapassou os $100 \%$ em decorrência de arredondamentos. Fonte: Dados da pesquisa (2020).

Foi encontrada associação entre a utilização do auxílio emergencial para a compra de fitas ou lancetas e a aquisição de insulinas $(\mathrm{p}=0,004)$, onde utilizar o auxílio para comprar fitas ou lancetas estava associado a comprar as insulinas com dinheiro próprio e inversamente associado a receber este insumo pelo SUS, em contrapartida, não utilizar o auxílio para adquirir tais insumos estava associado a adquirir insulinas por meio do SUS e inversamente associado a comprar insulinas com dinheiro próprio (Tabela 4).

Observou-se associação entre utilizar o auxílio emergencial para comprar fitas e lancetas e adquirir fitas reagentes no período antecedente a pesquisa $(<0,000)$, em que utilizar o auxílio para comprar fitas ou lancetas estava inversamente associado a adquirir fitas reagentes no SUS e associado a comprar esses insumos com recursos próprios ou doações. Todavia, não utilizar o auxílio para comprar fitas ou lancetas estava associado a receber tiras reagentes por meio do SUS e inversamente associado a comprar com dinheiro próprio ou doação (Tabela 4). 
Houve associação entre a utilização do auxílio emergencial para a compra de fitas ou lancetas e a aquisição usual de lancetas $(\mathrm{p}<0,000)$, onde o uso do auxílio estava associado a comprar lancetas com dinheiro próprio e inversamente associado a receber as lancetas por meio do SUS. Já a não utilização do auxílio para a aquisição destes insumos estava associada a receber as lancetas por meio do SUS e inversamente associada à compra com dinheiro próprio (Tabela 4).

Tabela 4. Associação entre o uso do auxílio emergencial para aquisição de materiais para manejo do diabetes e a forma adquirir insumos por adultos com Diabetes Tipo 1 no período de distanciamento social pela pandemia de COVID-19 no Brasil, 2020.

\begin{tabular}{|c|c|c|c|c|c|c|}
\hline \multirow{3}{*}{ Aquisição de Insumos } & \multicolumn{6}{|c|}{ Uso do Auxílio Emergencial } \\
\hline & \multicolumn{3}{|c|}{ Fitas e Lancetas } & \multicolumn{3}{|c|}{ Seringas ou Agulhas } \\
\hline & $\begin{array}{c}\text { Sim } \\
\mathrm{n}(\%)\end{array}$ & $\begin{array}{c}\text { Não } \\
\mathrm{n}(\%)\end{array}$ & p-valor* & $\begin{array}{c}\text { Sim } \\
\mathrm{n}(\%)\end{array}$ & $\begin{array}{c}\text { Não } \\
\text { n }(\%)\end{array}$ & p-valor* \\
\hline \multicolumn{7}{|l|}{ Insulinas } \\
\hline Pelo SUS & $14(7,5)^{(-)}$ & $88(47,3)^{(+)}$ & \multirow{6}{*}{$0,004 \mp$} & $7(3,8)^{(-)}$ & $109(58,6)$ & \multirow{6}{*}{$<0,000$ T } \\
\hline Dinheiro próprio & $21(11,3)^{(+)}$ & $34(18,3)^{(-)}$ & & $27(14,5)^{(+)}$ & $25(13,4)$ & \\
\hline Doação & $4(2,2)$ & $5(2,7)$ & & $4(2,2)^{(+)}$ & $1(0,5)$ & \\
\hline Não precisou adquirir & $3(1,6)$ & $7(3,8)$ & & 0 & $5(2,7)$ & \\
\hline Não consegui adquirir & $1(0,5)$ & 0 & & $1(0,5)$ & 0 & \\
\hline Outros & $2(1,1)$ & $7(3,8)$ & & $2(0,4)$ & $5(2,7)$ & \\
\hline \multicolumn{7}{|l|}{ Fitas Reagentes } \\
\hline Pelo SUS & $11(6)^{(-)}$ & $104(56,5)^{(+)}$ & \multirow{7}{*}{$<0,000$ Ф } & $11(7)$ & $89(56,3)$ & \multirow{7}{*}{0,056} \\
\hline Dinheiro próprio & $26(14,1)^{(+)}$ & $19(10,3)^{(-)}$ & & $13(8,2)$ & $27(17,1)$ & \\
\hline Doação & $5(2,7)^{(+)}$ & $4(2,2)^{(-)}$ & & $2(1,3)$ & $4(2,5)$ & \\
\hline Não precisou adquirir & $1(0,5)$ & $1(0,5)$ & & 0 & $1(0,6)$ & \\
\hline Não consegui adquirir & $2(1,1)$ & $3(1,6)$ & & $1(0,6)$ & $3(1,9)$ & \\
\hline Outros & 0 & $6(3,3)$ & & 0 & $5(3,2)$ & \\
\hline Não uso glicosímetro & 0 & $2(1,1)$ & & 0 & $2(1,3)$ & \\
\hline \multicolumn{7}{|l|}{ Lancetas } \\
\hline Pelo SUS & $10(5,4)^{(-)}$ & $90(48,9)^{(+)}$ & \multirow{7}{*}{$<0,000$ Ф } & $7(4,4)^{(-)}$ & $83(52,5)^{(+)}$ & \multirow{7}{*}{$0,004 T$} \\
\hline Dinheiro próprio & $23(12,5)^{(+)}$ & $22(12)^{(-)}$ & & $15(9,5)^{(+)}$ & $24(15,2)^{(-)}$ & \\
\hline Doação & $5(2,7)$ & $6(3,3)$ & & $2(1,3)$ & $6(3,8)$ & \\
\hline Não precisou adquirir & $6(3,3)$ & $10(5,4)$ & & $2(1,3)$ & $10(6,3)$ & \\
\hline Não consegui adquirir & 0 & $1(0,5)$ & & $0(0)$ & $1(0,6)$ & \\
\hline Outros & $1(0,5)$ & $7(3,8)$ & & $1(0,6)$ & $4(2,5)$ & \\
\hline Não uso glicosímetro & 0 & $3(1,6)$ & & $0(0)$ & $3(1,9)$ & \\
\hline \multicolumn{7}{|l|}{$S F M G^{* *}$} \\
\hline Pelo SUS & $1(0,6)$ & $4(2,4)$ & \multirow{7}{*}{0,568} & 0 & $5(3,5)$ & \multirow{7}{*}{0,266} \\
\hline Dinheiro próprio & $5(3)$ & $16(9,8)$ & & $2(1,4)$ & $18(12,7)$ & \\
\hline Doação & $3(1,8)$ & $3(1,8)$ & & $3(2,1)$ & $3(2,1)$ & \\
\hline Não precisou adquirir & 0 & $2(1,2)$ & & 0 & $2(1,4)$ & \\
\hline Não consegui adquirir & 0 & $1(0,6)$ & & 0 & $1(0,7)$ & \\
\hline Outros & $0(0)$ & $5(3)$ & & 0 & $3(2,1)$ & \\
\hline Não uso & $33(20,1)$ & $91(55,5)$ & & $20(14,1)$ & $85(59,9)$ & \\
\hline
\end{tabular}

*Teste de Qui-quadrado; **Sistema Flash de Monitorização da Glicemia; TEstatística significativa; Análise de resíduos: (+) Associação significativa, (-) Associação significativa negativa. Nota ${ }^{1}$ : $\mathrm{O}$ de participantes inferior na tabela em relação ao $\mathrm{n}$ total da pesquisa é em decorrência de alguns participantes não terem respondido essas perguntas no formulário. Nota $^{2}$ : A soma do percentual foi maior ou menor que $100 \%$ em decorrência de arredondamentos. Fonte: Dados da pesquisa (2020).

Verificou-se associação entre o uso do auxílio emergencial para a aquisição de seringas ou agulhas e aquisição de insulinas $(\mathrm{p}<0,000)$, onde usar o auxílio emergencial para comprar esses insumos foi associado a adquirir insulinas com dinheiro próprio ou doação, além de associação inversa com receber insulinas por meio do SUS. Foi encontrada também 
associação entre o uso do auxílio para comprar seringas ou agulhas e a aquisição de lancetas ( $\mathrm{p}=0,004)$, de modo que o uso do auxílio estava associado a comprar lancetas com dinheiro próprio e inversamente associado a receber lancetas por meio do SUS. Por outro lado, não utilizar o auxílio para adquirir seringas e agulhas estava associado a receber as lancetas por meio do SUS.

Foi encontrada associação entre a aquisição de insulinas ou hipoglicemiantes orais com o auxílio emergencial e a aquisição de insulinas $(\mathrm{p}<0,000)$. Observou-se que usar o auxílio emergencial para adquirir insulinas ou hipoglicemiantes orais estava associado a comprar insulinas com dinheiro próprio e a receber insulinas por doação, mas inversamente associado a receber insulinas por meio do SUS. Não utilizar o auxílio para adquirir insulinas e hipoglicemiantes estava associado a receber insulinas por meio do SUS e associado inversamente à compra com recursos financeiros próprios e doações (Tabela 5).

A aquisição de insulinas ou hipoglicemiantes por meio do auxílio emergencial e a aquisição de tiras reagentes demonstrou associação $(\mathrm{p}=0,004)$, observando-se que utilizar o auxílio para adquirir tais insumos estava associado inversamente a receber as fitas por meio SUS e associado a adquirir fitas reagentes com dinheiro próprio, por meio de doação ou a não ter conseguido adquirir as fitas. Não usar o dinheiro do auxílio para comprar insulinas ou hipoglicemiantes estava associado a receber fitas reagentes por meio do SUS e associado inversamente a comprar esses insumos por meio de recursos próprios e por doações (Tabela 5).

Foi encontrada associação entre o uso do auxílio emergencial para compra de insulinas e/ou hipoglicemiantes e a aquisição de lancetas ( $\mathrm{p}=0,001)$, onde foi observada associação entre usar o auxílio para adquirir insulinas ou hipoglicemiantes e realizar a compra de lancetas com dinheiro próprio e associação inversa entre adquirir lancetas por meio do SUS. Não usar o auxílio para comprar insulinas e hipoglicemiantes estava associado a receber as lancetas por meio do SUS e inversamente associado a adquirir com recursos próprios (Tabela 5).

O uso do auxílio para a compra de outros materiais relacionados ao tratamento do DM1 foi associado à aquisição de SFMG ( $\mathrm{p}=0,018$ ), demonstrando que adquirir outros materiais por meio do auxílio estava associado a adquirir SFMG por meio de dinheiro próprio e inversamente associado a não usar o SFMG. Por outro lado, não usar o auxílio para comprar outros materiais do tratamento estava inversamente associado a adquirir o SFMG com dinheiro próprio e associado a não usar essa tecnologia (Tabela 5). 
Tabela 5. Associação entre o uso do auxílio emergencial para compra de insulinas e/ou hipoglicemiantes e outros materiais relacionados ao diabetes com a aquisição de insumos por adultos com Diabetes Tipo 1 em período de distanciamento social pela pandemia de COVID-19 no Brasil, 2020.

\begin{tabular}{|c|c|c|c|c|c|c|}
\hline \multirow{3}{*}{ Aquisição de Insumos } & \multicolumn{6}{|c|}{ Uso do Auxílio Emergencial } \\
\hline & \multicolumn{3}{|c|}{ Insulina ou hipoglicemiantes } & \multicolumn{3}{|c|}{ Outros Materiais DM } \\
\hline & $\begin{array}{c}\text { Sim } \\
\mathrm{n}(\%)\end{array}$ & $\begin{array}{c}\text { Não } \\
\mathrm{n}(\%)\end{array}$ & p-valor* & $\begin{array}{c}\mathrm{Sim} \\
\mathrm{n}(\%)\end{array}$ & $\begin{array}{c}\text { Não } \\
\mathrm{n}(\%)\end{array}$ & p-valor* \\
\hline \multicolumn{7}{|l|}{ Insulinas } \\
\hline Pelo SUS & $9(4,8)^{(-)}$ & $93(50)^{(+)}$ & \multirow{6}{*}{$<0,000 \Phi$} & $18(9,7)$ & $84(45,2)$ & \multirow{6}{*}{0,142} \\
\hline Dinheiro próprio & $23(12,4)^{(+)}$ & $32(17,2)^{(-)}$ & & $16(8,6)$ & $39(21)$ & \\
\hline Doação & $5(2,7)^{(+)}$ & $4(2,2)^{(-)}$ & & $3(1,6)$ & $6(3,2)$ & \\
\hline Não precisou adquirir & $1(0,5)$ & $9(4,8)$ & & $1(0,5)$ & $9(4,8)$ & \\
\hline Não conseguiu adquirir & $1(0,5)$ & 0 & & $1(0,5)$ & 0 & \\
\hline Outros & $2(1,1)$ & $7(3,8)$ & & $3(1,6)$ & $6(3,2)$ & \\
\hline \multicolumn{7}{|l|}{ Fitas Reagentes } \\
\hline Pelo SUS & $16(8,7)^{(-)}$ & $99(53,8)^{(+)}$ & \multirow{7}{*}{$0,004 \mathrm{~T}$} & $19(10,3)$ & $96(52,2)$ & \multirow{7}{*}{0,117} \\
\hline Dinheiro próprio & $15(8,2)^{(+)}$ & $30(16,3)^{(-)}$ & & $15(8,2)$ & $30(16,3)$ & \\
\hline Doação & $5(2,7)^{(+)}$ & $4(2,2)^{(-)}$ & & $4(2,2)$ & $5(2,7)$ & \\
\hline Não precisou adquirir & 0 & $2(1,1)$ & & $1(0,5)$ & $1(0,5)$ & \\
\hline Não consegui adquirir & $3(1,6)^{(+)}$ & $2(1,1)$ & & $2(1,1)$ & $3(1,6)$ & \\
\hline Outros & $2(1,1)$ & $4(2,2)$ & & $1(0,5)$ & $5(2,7)$ & \\
\hline Não uso glicosímetro & 0 & $2(1,1)$ & & 0 & $2(1,1)$ & \\
\hline \multicolumn{7}{|l|}{ Lancetas } \\
\hline Pelo SUS & $11(6)^{(-)}$ & $89(48,4)^{(+)}$ & \multirow{7}{*}{$0,001 \mathrm{~T}$} & $17(9,2)$ & $83(45,1)$ & \multirow{7}{*}{0,282} \\
\hline Dinheiro próprio & $16(8,7)^{(+)}$ & $29(15,8)^{(-)}$ & & $16(8,7)$ & $29(15,8)$ & \\
\hline Doação & $5(2,7)$ & $6(3,3)$ & & $3(1,6)$ & $8(4,3)$ & \\
\hline Não precisou adquirir & $6(3,3)$ & $10(5,4)$ & & $4(2,2)$ & $12(6,5)$ & \\
\hline Não consegui adquirir & $1(0,5)$ & 0 & & 0 & $1(0,5)$ & \\
\hline Outros & $2(1,1)$ & $6(3,3)$ & & $2(1,1)$ & $6(3,3)$ & \\
\hline Não uso glicosímetro & 0 & $3(1,6)$ & & 0 & $3(1,6)$ & \\
\hline \multicolumn{7}{|l|}{$S F M G^{* *}$} \\
\hline Pelo SUS & 0 & $5(3)$ & & $2(1,2)$ & $3(1,8)$ & \multirow{7}{*}{0,018 T } \\
\hline Dinheiro próprio & $6(3,7)$ & $15(9,1)$ & & $10(6,1)^{(+)}$ & $11(6,7)^{(-)}$ & \\
\hline Doação & $2(1,2)$ & $4(2,4)$ & & $3(1,8)$ & $3(1,8)$ & \\
\hline Não precisou adquirir & 0 & $2(1,2)$ & 0,776 & 0 & $2(1,2)$ & \\
\hline Não consegui adquirir & 0 & $1(0,6)$ & & 0 & $1(0,6)$ & \\
\hline Outros & $1(0,6)$ & $4(2,4)$ & & $3(1,8)$ & $2(1,2)$ & \\
\hline Não uso & $27(16,5)$ & $97(59,1)$ & & $23(14)^{(-)}$ & $101(61,6)^{(+)}$ & \\
\hline
\end{tabular}

*Teste de Qui-quadrado; **Sistema Flash de Monitorização da Glicemia; TEstatística significativa; Análise de resíduos: (+) Associação significativa, (-) Associação significativa negativa. Nota ${ }^{1}$ : $\mathrm{n}$ de participantes inferior na tabela em relação ao $\mathrm{n}$ total da pesquisa é em decorrência de alguns participantes não terem respondido essas perguntas no formulário. Nota ${ }^{2}$ : A soma do percentual foi maior ou menor que $100 \%$ em decorrência de arredondamentos. Fonte: Dados da pesquisa (2020).

\section{Discussão}

Este estudo caracterizou o recebimento do auxílio emergencial e analisou o uso deste por adultos com DM1 durante a pandemia de COVID-19 no Brasil. A associação entre ter recebido o auxílio emergencial e ter renda familiar de até 2 SM estava de acordo com os critérios para recebimento do benefício, no qual a renda familiar total deve ser de até 3 SM (Brasil, 2020).

Os resultados indicaram que pertencer às classes sociais mais baixas estava associado ao recebimento do auxílio emergencial e sabe-se que menores níveis socioeconômicos (considerando renda, classe social e escolaridade) têm sido associados a um pior manejo da glicemia (Scott et al., 2017), o que pode ser um fator de risco para o aumento na morbimortalidade de pacientes com diabetes e COVID-19 (Chen et al., 2020; Holman et al., 2020). 
Nesse contexto, apesar do SARS-COV-2 não ter seletividade quanto ao contágio, os impactos diretos e indiretos da pandemia são sentidos de formas diferentes em função da raça, classe e gênero, muito em decorrência das desigualdades sociais exacerbadas durante este período (Estrela et al., 2020). Este cenário demonstra que o auxílio financeiro poderia garantir melhores condições de saúde (Magalhães et al., 2013), amenizando o impacto econômico de medidas de distanciamento social nas famílias (Mackee \& Stuckler, 2020).

Este estudo mostrou que o auxílio emergencial foi utilizado principalmente para o pagamento de contas e aquisição de medicamentos pelos participantes, demonstrando a importância deste para cobrir gastos básicos. Não existem dados oficiais sobre como os brasileiros têm utilizado o auxílio emergencial, porém, segundo dados da pesquisa Datafolha, mais de 50\% dos beneficiários usaram o auxílio para comprar alimentos, seguido do pagamento de contas (25\%), como internet, e pagamento das despesas de casa (26\%) (Datafolha, 2020).

Segundo a POF (2017-2018) (IBGE, 2019), mesmo antes da pandemia, o Brasil já havia apresentado um aumento da insegurança alimentar em relação às pesquisas anteriores, apontando algum grau de incerteza em relação à regularidade do acesso aos alimentos. Este cenário está sendo potencializado com a pandemia de COVID-19 (Oliveira et al., 2020), portanto, o auxílio emergencial torna-se uma estratégia fundamental para minimizar o impacto da pandemia na segurança alimentar (Gurgel et al., 2020).

Para Alpino et al., (2020), a pandemia por COVID-19 afeta principalmente os indivíduos mais vulneráveis, pois apresenta influência na oferta e demanda dos alimentos, diminui o poder de compra e a capacidade de produção e distribuição. Os autores destacam ainda que todas as ações propostas pelo Governo Federal para atenuar os efeitos da pandemia consistem em deliberações que se atentam em especial ao acesso à renda e aos alimentos. Todavia, entende-se que para garantir o Direito Humano à Alimentação Adequada e a Segurança Alimentar e Nutricional, exigem-se medidas focadas não apenas em diminuir as consequências da crise, mas também em medidas que a médio e longo prazo garantam o direito constitucional à alimentação adequada.

Foi possível observar que usar o auxílio emergencial para comprar insumos estava associado ao uso de dinheiro próprio ou doação para este fim. Em contrapartida, não usar o auxílio emergencial para a compra de insumos estava associado a conseguir adquirir os insumos por meio do SUS. Sugere-se que aqueles indivíduos que compravam seus insumos com dinheiro próprio mantiveram esta prática durante a pandemia, entretanto, possivelmente utilizando o valor referente ao auxílio emergencial, o que simboliza que um valor que poderia ser usado para compensar possíveis reduções na renda familiar e custear necessidades básicas acaba sendo destinado para a compra de insumos, os quais deveriam ser distribuídos regularmente pelo SUS, de acordo com a Lei No 11.347/2006 (Brasil, 2006).

Ressalta-se que o DM1 é uma condição crônica que requer cuidados diários, o que demanda grande quantidade de insumos para uso contínuo durante toda a vida (SBD, 2019). O custo desse manejo impacta no orçamento familiar e, quando não há fornecimento regular dos insumos pelo SUS, ocorre um aumento nos gastos com o tratamento que são retirados do orçamento familiar desses indivíduos (Ogle et al., 2016). Visando a redução de custos, os pacientes com baixo poder aquisitivo podem reduzir doses de insulinas, reutilizar produtos descartáveis e realizar a automonitorização glicêmica em frequência inferior ao recomendado, o que pode impactar no controle glicêmico, desenvolvimento de complicações micro e macrovasculares (Cobas et al., 2013) e pior desfecho em quadros de COVID-19 (Chen et al., 2020; Holman et al., 2020).

Um estudo realizado na Jordânia com 229 famílias de crianças e adolescentes com DM1 identificou que o lockdown, em decorrência da pandemia por COVID-19, impactou a disponibilidade de medicamentos, incluindo a insulina, além de tiras reagentes. Quase dois terços das famílias estudadas realizavam a compra de insulinas com dinheiro próprio e precisaram racionar as doses de medicamentos e insulina, além de 43,5\% das famílias terem realizado racionamento de tiras reagentes, das quais, 75,5\% tiveram hipoglicemias e hiperglicemias associadas a essa prática (Odeh et al., 2020). 
Verma et al., (2020), também identificaram problemas no acesso às insulinas e tiras reagentes, o que impactou negativamente o controle glicêmico dos pacientes com diabetes durante a pandemia. Os autores enfatizaram que as disparidades na saúde relacionadas ao nível socioeconômico, as quais já existiam antes da pandemia, foram exacerbadas neste momento de calamidade pública.

Portanto, considera-se que garantir a oferta regular de insumos por meio do SUS pode evitar que as pessoas com DM1 dependam do auxílio emergencial para a compra desses materiais e consigam suprir outras necessidades básicas, como a alimentação, podendo atuar como uma proteção ao indivíduo e família.

Neste estudo, não usar o SFMG estava associado ao recebimento do auxílio emergencial. É possível que o alto custo de aquisição do SFMG no Brasil demonstre claramente uma diferença de acesso às tecnologias para o tratamento do DM1 entre pessoas de diferentes classes sociais.

Por fim, os resultados reforçam achados já discutidos por outros estudos sobre as políticas públicas implementadas durante a Pandemia de COVID-19. Filho e Júnior, (2020) afirmam que, durante a pandemia, as ações do Governo Federal estão aquém das dificuldades que a população brasileira mais pobre está enfrentando, sendo assim, o valor do auxílio emergencial não supre as necessidades básicas da população. A urgência da pandemia requer a adoção de políticas públicas de emprego e programas de transferência de renda que visem proteger os trabalhadores, em especial do setor informal, os mais atingidos economicamente pela pandemia (Costa, 2020). Em um contexto pós-pandemia, Costa (2020) sugere que o governo eleve os gastos com programas sociais e programas de transferência de renda, o que, inevitavelmente, irá ampliar o déficit público, mas que serão medidas imprescindíveis para o enfrentamento da pobreza, principalmente entre os mais vulneráveis.

Por isso, estudos como este são de extrema importância a fim de que se identifiquem as características da população com DM1 que recebeu o auxílio emergencial e como utilizou tal benefício, para que se planeje políticas públicas a serem implementadas em longo prazo. Destaca-se o caráter inédito deste estudo, visto que não foram encontradas outras pesquisas que façam descrições semelhantes tanto a nível nacional como internacional.

Como limitações do presente estudo, a pesquisa na modalidade online pode avaliar apenas um recorte na população, excluindo os indivíduos que não têm acesso regular aos meios eletrônicos ou à internet. Além disso, não foram analisadas variáveis referentes ao tipo de trabalho (formal ou informal) dos sujeitos da pesquisa, sendo importante incluir a análise desta variável em pesquisas futuras.

\section{Conclusão}

Foi possível observar que os participantes utilizaram o auxílio emergencial principalmente para o pagamento de contas básicas e compra de alimentos. O uso do auxílio emergencial para comprar insumos do tratamento estava associado a comprá-los com dinheiro próprio ou recebê-los por doação, enquanto não usar o auxílio para a compra de insumos estava associado a adquiri-los por meio do SUS. Portanto, o presente estudo reforça a necessidade de políticas públicas que possam amenizar as desigualdades de acesso à saúde, especialmente em uma situação de calamidade pública, como a Pandemia de COVID-19.

\section{Referências}

Alpino, T. de M. A., Santos, C. R. B., Barros, D. C. de, \& Freitas, C. M. de. (2020). COVID-19 and food and nutritional (in)security: action by the Brazilian Federal Government during the pandemic, with budget cuts and institutional dismantlement. Cadernos de Saude Publica, 36(8). https://doi.org/10.1590/0102$311 \mathrm{X} 00161320$

Andrade, C. J. do N., \& Alves, C. de A. D. (2014). Análise comparativa do controle glicêmico de crianças com diabetes melito tipo 1 com base na distribuição de insumos : capital x interior da Bahia. Rev. Ciênc. Méd. Biol, 13(3), 274-279.

Arrais, T. A., Oliveira, A. R. de, Alencar, D. P., Salgado, T. R., Viana, J. L., \& Miranda, A. F. (2020). Pandemia covid-19: o caráter emergencial das 
transferências de renda direta e indireta para a população vulnerável do estado de Goiás. Espaço e Economia, 18 . https://doi.org/10.4000/espacoeconomia.13734

Barreto, M. L., Barros, A. J. D. de, Carvalho, M. S., Codeço, C. T., Hallal, P. R. C., Medronho, R. de A., Struchiner, C. J., Victora, C. G., \& Werneck, G. L. (2020). O que é urgente e necessário para subsidiar as políticas de enfrentamento da pandemia de COVID-19 no Brasil? Rev Bras Epidemiol, $23,1-4$. https://doi.org/10.1590/1980-549720200032

Brasil (2020). Lei No 13.982, de 2 de abril de 2020. Diário Oficial da União 2020; 02 mar. https://www.in.gov.br/en/web/dou/-/lei-n-13.982-de-2-de-abril-de2020-250915958

Brasil (2021). Decreto No 10.661, de 26 de março de 2021. Diário Oficial da União 2021; 26 mar. https://www.in.gov.br/en/web/dou/-/decreto-n-10.661-de26-de-marco-de-2021-310836042

Brasil (2006). Lei N 11.347 de 27 de setembro de 2006. Diário Oficial da União 2006; 28 set. http://www.planalto.gov.br/ccivil_03/_ato20042006/2006/lei/111347.htm

Brasil (2012). Resolução Nº 466, de 12 de dezembro de 2012. http://conselho.saude.gov.br/resolucoes/2012/Reso466.pdf

Brasil (2016). Resolução No 510, de 7 de abril de 2016. https://www.in.gov.br/materia///asset_publisher/Kujrw0TZC2Mb/content/id/22917581.

Carvalho, André Roncaglia de et al. Vulnerabilidade social e crise sanitária no Brasil (2021). Cadernos de Saúde Pública [online]. v. 37, n. 9, e00071721. <https://doi.org/10.1590/0102-311X00071721>.

Chen, J., Wu, C., Wang, X., Yu, J., \& Sun, Z. (2020). The Impact of COVID-19 on Blood Glucose : A Systematic Review and Meta-Analysis. Frontiers in Endocrinology, 11(October), 1-8. https://doi.org/10.3389/fendo.2020.574541

Cobas, R. A., Ferraz, B., Mattos, S. De, Righeti, L., Tannus, M., Negrato, A., Araujo, A. De, Dib, A., \& Brito, M. (2013). The cost of type 1 diabetes : a nationwide multicentre study in Brazil. Bull World Health Organ, 91, 434-440. https://doi.org/http://dx.doi.org/10.2471/BLT.12.110387

Córdova, I. de, \& Alves, I. G. (2019). Programas de transferência condicionada de renda na ótica dos direitos sociais: uma opção para o combate das vulnerabilidades brasileiras. Polis, 53. http://journals.openedition.org/polis/17550

Costa, S. da S. (2020). Pandemia e desemprego no Brasil. Revista de Administração Pública, 54(4), 969-978. https://doi.org/10.1590/0034-761220200170 Datafolha (2020). Auxílio emergencial: maioria dos brasileiros usa benefício para comprar comida. Infomoney, 14 ago. 2020. <https://www.infomoney.com.br/minhas-financas/auxilio-emergencial-maioria-dos-brasileiros-usa-beneficio-para-comprar-comida-diz-datafolha/>.

Estrela, F. M., Soares, C. F. S. E., Cruz, M. A. da, Silva, A. F. da, Santos, J. R. L., Moreira, T. M. de O., Lima, A. B., \& Silva, M. G. (2020). Pandemia da Covid 19: refletindo as vulnerabilidades a luz do gênero, raça e classe. Ciencia \& Saude Coletiva, 25(9), 3431-3436. https://doi.org/10.1590/141381232020259.14052020

Filho, O. J. da S., \& Júnior, N. N. G. (2020). O amanhã vai à mesa: abastecimento alimentar e COVID-19. Cadernos de Saúde Pública, 36(5). https://doi.org/10.1590/0102-311X00095220

Gurgel, A. do M., Alves, K. P. de S., Araujo, J. M. de, \& Leal, V. S. (2020). Estratégias governamentais para a garantia do direito humano à alimentação adequada e saudável no enfrentamento à pandemia de Covid-19 no Brasil. Ciência \& Saúde Coletiva, Set. http://www.cienciaesaudecoletiva.com.br/artigos/estrategias-governamentais-para-a-garantia-do-direito-humano-a-alimentacao-adequada-e-saudavel-noenfrentamento-a-pandemia-de-covid19-no-brasil/17778?id=17778

Holman, N., Knighton, P., Kar, P., O'Keefe, J., Curley, M., Weaver, A., Barron, E., Bakhai, C., Khunti, K., Wareham, N. J., Sattar, N., Young, B., \& Valabhji, J. (2020). Risk factors for COVID-19-related mortality in people with type 1 and type 2 diabetes in England: a population-based cohort study. The Lancet Diabetes and Endocrinology, 8(10), 823-833. https://doi.org/10.1016/S2213-8587(20)30271-0

International Diabetes Federation - IDF. (2021). IDF Diabetes Atlas 10th edition. https://diabetesatlas.org/idfawp/resourcefiles/2021/07/IDF_Atlas_10th_Edition_2021.pdf

Instituto Brasileiro de Geografia e Estatística - IBGE. (2019). Pesquisa de Orçamentos Familiares 2017-2018. https://biblioteca.ibge.gov.br/visualizacao/livros/liv101670.pdf

Instituto Brasileiro de Geografia e Estatística (2021). Séries históricas da taxa de desemprego. https://www.ibge.gov.br/explica/desemprego.php Magalhães, K. A., Cotta, R. M. M., Martins, T. de C. P., \& Siqueira-Batista, A. P. G. R. (2013). A Habitação como Determinante Social da Saúde : percepções e condições de vida de famílias cadastradas no Programa Bolsa Família. Saúde Soc. São Paulo, 22(1), 57-72.

Mckee, M., \& Stuckler, D. (2020). If the world fails to protect the economy, COVID-19 will damage health not just now but also in the future. Nature Medicine, 26(May), 640-642. https://doi.org/10.1038/s41591-020-0863-y

Ministério da Saúde (2007). Portaria No 2.583 de 10 de outubro de 2007. http://bvsms.saude.gov.br/bvs/saudelegis/gm/2007/prt2583_10_10_2007.html. Odeh, R., Gharaibeh, L., Daher, A., Kussad, S., \& Alassaf, A. (2020). Caring for a child with type 1 diabetes during COVID-19 lockdown in a developing country: Challenges and parents' perspectives on the use of telemedicine. Diabetes Research and Clinical Practice, 168(January).

Ogle, G. D., Kim, H., Middlehurst, A. C., Silink, M., \& Jenkins, A. J. (2016). Financial costs for families of children with Type 1 diabetes in lower-income countries. Diabetic Medicine, 33(6), 820-826. https://doi.org/10.1111/dme.12997

Oliveira, T. C., Abranches, M. V., \& Lana, R. M. (2020). (In)Segurança alimentar no contexto da pandemia por SARS-CoV-2. Cadernos de Saude Publica, 36(4), 1-6. https://doi.org/10.1590/0102-311X00055220

Sauza, V. P. de, Santos, E. C. B. dos, Angelim, R. C. de M., Teixeira, C. R. de S., \& Martins, R. D. (2018). Conhecimento e Práticas de Usuários com Diabetes Mellitus Sobre a Automonitorização da Glicemia Capilar no Domicilio. J. Res.: Fundam. Care. Online, 10(3), 737-745. https://doi.org/10.9789/2175-5361.2018.v10i3.737-745 
Research, Society and Development, v. 11, n. 2, e55211225907, 2022

(CC BY 4.0) | ISSN 2525-3409 | DOI: http://dx.doi.org/10.33448/rsd-v11i2.25907

Scott, A., Chambers, D., Goyder, E., \& O’Cathain, A. (2017). Socioeconomic inequalities in mortality, morbidity and diabetes management for adults with type 1 diabetes: A systematic review. PLoS ONE, 12(5), 1-19. https://doi.org/10.1371/journal.pone.0177210

Sociedade Brasileira de Diabetes. (2019). Diretrizes Sociedade Brasileira de Diabetes 2019-2020 (Clanad Editora Científica (ed.)). https://www.diabetes.org.br/profissionais/images/DIRETRIZES-COMPLETA-2019-2020.pdf

Verma, A., Rajput, R., Verma, S., Balania, V. K. B., \& Jangra, B. (2020). Impact of lockdown in COVID 19 on glycemic control in patients with type 1 Diabetes Mellitus. Diabetes and Metabolic Syndrome: Clinical Research and Reviews, 14(5), 1213-1216. https://doi.org/10.1016/j.dsx.2020.07.016 